\title{
The impact of transferring patients with ST-segment elevation myocardial infarction to percutaneous coronary intervention-capable hospitals on clinical outcomes
}

Bo Won Kim ${ }^{1}$, Kwang Soo Cha ${ }^{1}$, Min Joung Park ${ }^{1}$, Jong Hyun Choi ${ }^{1}$, Eun Young Yun ${ }^{1}$, Jin Sup Park ${ }^{1}$, Hye Won Lee ${ }^{1}$, Jun-Hyok Oh ${ }^{1}$, Jeong Su Kim ${ }^{2}$, Jung Hyun Choi ${ }^{1}$, Young Hyun Park ${ }^{2}$, Han Cheol Lee ${ }^{1}$, June Hong Kim², Kook Jin Chun², Taek Jong Hong ${ }^{1}$, Youngkeun $\mathrm{Ahn}^{3}$, Myung Ho Jeong ${ }^{3}$; the Korea Acute Myocardial Infarction Registry Investigators

${ }^{1}$ Pusan National University Hospital, Busan, South Korea

${ }^{2}$ Pusan National University Yangsan Hospital, Yangsan, South Korea

${ }^{3}$ Chonnam National University Hospital, Gwangju, South Korea

\section{Abstract}

Background: Primary percutaneous coronary intervention (PCI) is recommended for ST-segment elevation myocardial infarction (STEMI) patients even when the patient must be transported to a PCI-capable hospital. This study aimed to evaluate the long-term clinical outcomes of STEMI patients who were transferred for primary PCI compared to patients who arrived directly to PCI-capable hospitals.

Methods: A total of 3,576 STEMI patients with less than 12 h of symptom onset-to-door time from the Korea Acute Myocardial Infarction Registry were divided into transfer $(n=2,176)$ and direct-arrival $(n=1,400)$ groups according to their status. The primary outcome was the composite of major adverse cardiac event (MACE), defined as death, non-fatal myocardial infarction, and revascularization at 1 year.

Results: In the transfer $v$ s. the direct-arrival group, the median symptom onset-to-firstmedical contact time was significantly shorter (60 vs. $80 \mathrm{~min}, p<0.001$ ), but the median symptom onset-to-door time was significantly longer (194 vs. $90 \mathrm{~min}, p<0.001$ ). The median door-toballoon time was significantly shorter in the transfer group vs. the direct-arrival group (75 vs. $91 \mathrm{~min}, p<0.001)$. Total death and the composite of MACE were not significantly different during hospitalization (5.1 vs. 3.9\%, $p=0.980 ; 5.4$ vs. $4.8 \%, p=0.435$, respectively) and at 1 -year (8.2 vs. $6.6 \%, p=0.075 ; 13.7$ vs. $13.9 \%, p=0.922$, respectively).

Conclusions: Transferring STEMI patients to PCI-capable hospitals with a time delay did not affect clinical outcomes after 1 year. This study suggests that inter-hospital transfer should be encouraged even with delay for STEMI patients who require primary PCI in areas with a similar geographic accessibility. (Cardiol J 2016; 23, 3: 289-295)

Keywords: transfer, myocardial infarction, comparative effectiveness research, percutaneous coronary intervention

Address for correspondence: Kwang Soo Cha, MD, PhD, Department of Cardiology and Medical Research Institute, Pusan National University Hospital, 179 Gudeok-ro Seo-gu, Busan 602-739, South Korea, tel: +82-51-240-7221, fax: +82-51-247-5875, e-mail: chakws1@hanmail.net,chakws@gmail.com 


\section{Introduction}

Rapid reperfusion by primary percutaneous coronary intervention (PCI) is the preferred strategy for treating patients with ST-segment elevation myocardial infarction (STEMI) [1]. Guidelines recommend that reperfusion therapy with primary PCI should be administered as soon as possible to all eligible patients with STEMI. Delays in door-toballoon times are associated with increased mortality [2-4]. Guidelines recommend a door-to-balloon time of $90 \mathrm{~min}$ or less [5,6]. Unfortunately, in the United States, only $25 \%$ of hospitals are capable of performing PCI [7]. Therefore, if a patient with STEMI arrives at a non-PCI-capable hospital, they should be transferred to a PCI-capable hospital for primary PCI if the anticipated first medical contactto-device time at that hospital does not exceed 120 min $[5,6]$. In fact, only $12 \%$ of transferred patients were treated with PCI within 90 min of the time from the first medical contact [8]. Therefore, patient transfer can be a cause of significantly delayed treatment.

However, several studies have shown that pre-hospital electrocardiographic diagnosis and direct referral to PCI-capable hospitals decrease the time delay in treating patients with STEMI $[1,8,9]$. Quality improvement strategies have reduced door-to-balloon times for direct-arrival patients with STEMI undergoing primary PCI. Nevertheless, bypassing the local hospital is not always realistic, and many STEMI patients are still required to go through inter-hospital transfer for primary PCI.

We examined patients with STEMI who were registered in the Korea Acute Myocardial Infarction Registry (KAMIR). The aim of this study was to assess the clinical differences between transferred patients and direct-arrival patients. In addition, we aimed to evaluate the long-term clinical outcomes of patients with STEMI that were either transferred for primary PCI or direct-arrival patients.

\section{Methods}

\section{Study population and study design}

We analyzed data from the KAMIR database. KAMIR is a prospective, multicenter, observational online registry designed to survey the epidemiology, management, clinical features, and prognosis of acute myocardial infarction (MI) patients in Korea, and it is supported by the Korean Circulation Society [10, 11]. A total of 50 hospitals in Korea are enrolled in KAMIR. These hospitals are high- volume centers with facilities for PCI and on-site cardiac surgery. This study was approved by the ethics committee at each participating institution. All patients provided informed consent to participate before enrollment.

A total of 14,885 consecutive patients admitted between November 2005 and July 2007 with acute MI were included in the KAMIR, and 13,003 patients were confirmed as having MI at discharge. However, 4,516 patients were excluded due to missing or invalid symptom-to-door times or invalid door-to-balloon times. Among the remaining 8,487 patients, 5,650 patients were diagnosed with STEMI and 2,837 patients were diagnosed with non-STEMI. Of the STEMI patients, 2,068 patients were excluded due to a symptom-to-door time $>12 \mathrm{~h}$, a symptom-to-first-medical-contact time $>12 \mathrm{~h}$, or a door-to-balloon time $>6 \mathrm{~h}$. In addition, data on 6 patients were missing. Therefore, 3,582 patients were included in the final study population, which was divided into two groups: Group 1: those who were transferred to a PCI-capable hospital $(\mathrm{n}=2,176)$ and Group 2: those who arrived directly at a PCI-capable hospital $(\mathrm{n}=1,400)$. A flow chart of study population selection is presented in Figure 1. In this study, we analyzed baseline characteristics and then studied angiographic findings, mortality, and morbidity for 1 year.

The diagnosis of acute MI was based on clinical presentation, electrocardiographic findings, and levels of cardiac enzymes. Patients were diagnosed with STEMI when they had new, or presumed new, ST-segment elevation of at least $1 \mathrm{~mm}$ seen in any location, a new left bundle-branch block on the index, or a subsequent electrocardiogram (ECG) with at least 1 positive cardiac biochemical marker of necrosis (including creatine kinase-MB and troponins I and T) [12].

\section{Statistical analysis}

Data are expressed as mean \pm standard deviation for continuous variables and percentages for categorical variables. Differences in the means of continuous measurements were tested by either the Student's t-test or ANOVA. Analyses of discrete variables were performed using the $\chi^{2}$ test. All comparison between baseline characteristics was assessed with the ANOVA for continuous variables, and with the $\chi^{2}$ test for categorical variables. For clinical outcomes, an F-test was performed to test whether major adverse cardiac events (MACE, percentage) between the transfer and direct-arrival groups were different. In-hospital, 1-, 6-, and 12-month MACE were evaluated by comparing the 


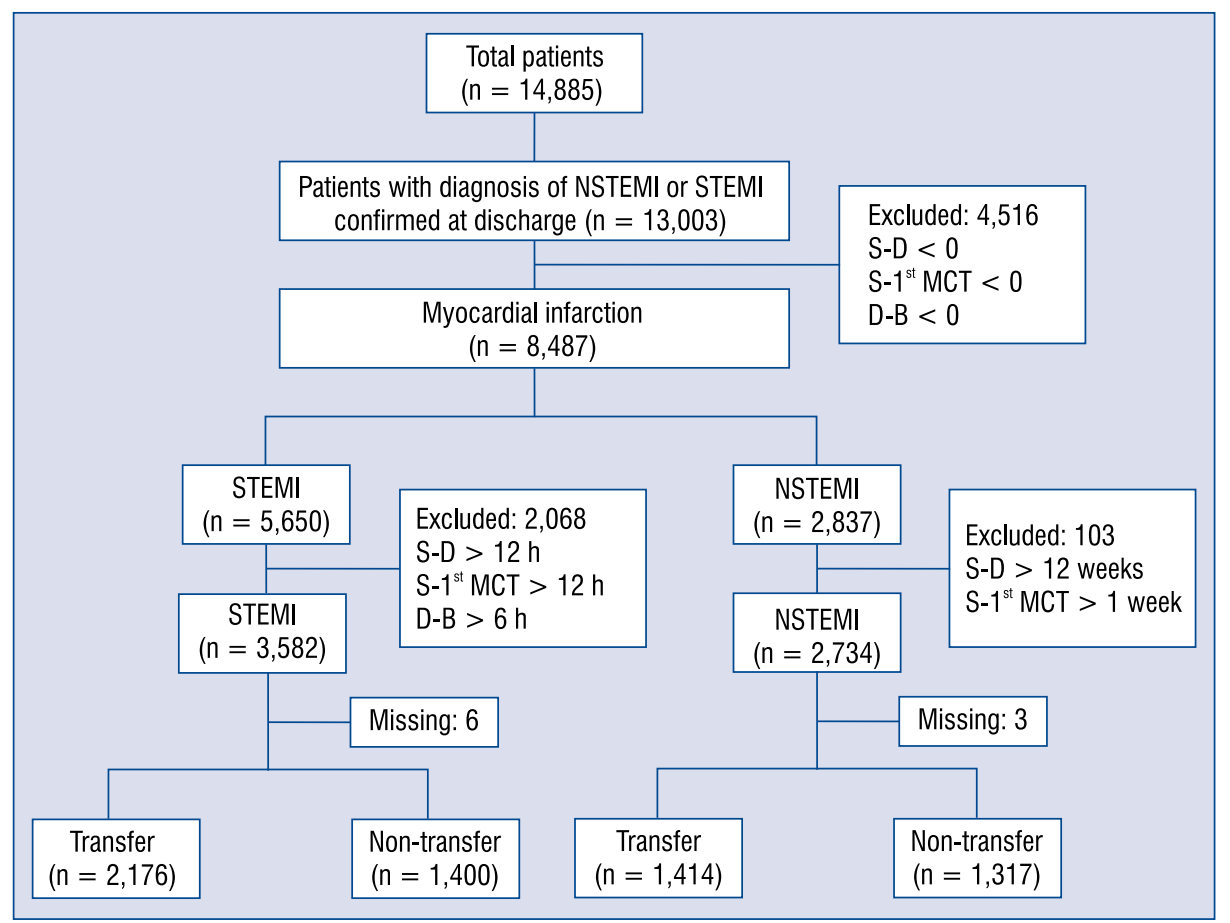

Figure 1. Flow chart of the study population selection. A total of 13,003 patients were confirmed as ST-segment elevation myocardial infarction (STEMI) or non-STEMI (NSTEMI) at discharge and recruited for this study. However, 4,516 patients were excluded due to a symptom-to-door time $(\mathrm{S}-\mathrm{D})<0$, symptom-to-first medical contact time $\left(\mathrm{S}-1^{\text {st }} \mathrm{MCT}\right)<0$, door-to-balloon time (D-B) < 0, or symptom-door-to-first medical contact $<0$. Out of the remaining 8,487 patients, 5,650 patients were STEMI patients and 2,837 patients were NSTEMI patients. In STEMI patients, 2,068 patients were excluded because of a symptom-to-door time $>12 \mathrm{~h}$, symptom-to-first medical contact time $>12 \mathrm{~h}$, door-to-balloon time $>6 \mathrm{~h}$, and 6 were missing. Ultimately, 3,582 patients were divided into two groups based on their status as transfer or non-transfer.

mean values (percentage) using ANOVA. A value of $p<0.05$ was considered significantly significant.

\section{Results}

\section{Baseline patient characteristics}

The 3,576 patients with STEMI who underwent primary PCI between November 2005 and July 2007 were divided into two groups. Of these patients, 2,176 (60.9\%), who arrived at a non-PCI-capable hospital and were transferred to a PCI-capable hospital, were assigned to the transfer group. A group of 1,400 (39.1\%) patients who arrived directly to PCI-capable hospital were assigned to the direct-arrival group. There were some differences in the baseline patient characteristics between the transfer and direct-arrival groups. Compared to the direct-arrival group, transfer group patients were more likely to be older and male. In addition, $43 \%$ of the transferred patients had hypertension, $23 \%$ had diabetes, and $7 \%$ had dyslipidemia. In the direct-arrival group, $42 \%$ of patients had hypertension, $24 \%$ had diabetes, and $10 \%$ had dyslipidemia.
There was a statistically significant difference in all of these parameters (Table 1 ). In contrast, there was no statistically significant difference in other parameters, such as smoking status or body mass index (Table 1).

\section{Treatment delay}

Treatment delay parameters are shown in Figure 1. Over the 2-year study period, the patients in the transfer group had shorter symptom onset-to-first medical contact times compared to patients in the direct-arrival group (60 vs. $80 \mathrm{~min}, \mathrm{p}<0.001$ ). Also, the door-to-balloon time was significantly shorter in patients in the transfer group compared to those in the direct-arrival group (75 vs. $91 \mathrm{~min}$, $\mathrm{p}<0.001)$. However, patients in the transfer group had a significantly longer symptom onset-to-door time at PCI-capable centers compared to direct-arrival patients (194 vs. $90 \mathrm{~min}, \mathrm{p}<0.001$ ). In addition, the symptom onset-to-balloon time was significantly longer for patients in the transfer group compared to the direct-arrival group (283 vs. $205 \mathrm{~min}, \mathrm{p}<0.001$ ). 
Table 1. Baseline characteristics.

\begin{tabular}{|c|c|c|c|}
\hline Variable & $\begin{array}{l}\text { Transfer group } \\
(n=2,176)\end{array}$ & $\begin{array}{l}\text { Direct-arrival group } \\
(\mathrm{n}=1,400)\end{array}$ & $\mathbf{P}$ \\
\hline Age [year] & $61.83 \pm 12.7$ & $60.76 \pm 12.81$ & 0.014 \\
\hline Male sex & $599(27.55 \%)$ & $322(23.02 \%)$ & 0.002 \\
\hline Body mass index $\left[\mathrm{kg} / \mathrm{m}^{2}\right]$ & $23.96 \pm 3.35$ & $24.08 \pm 3.23$ & 0.290 \\
\hline \multicolumn{4}{|l|}{ Risk factors } \\
\hline Hypertension & $938(43.1 \%)$ & $659(41.7 \%)$ & 0.044 \\
\hline Diabetes mellitus & $499(22.9 \%)$ & $331(23.6 \%)$ & 0.046 \\
\hline Dyslipidemia & $162(7.4 \%)$ & $142(10.1 \%)$ & 0.017 \\
\hline Smoking & $1375(63.4 \%)$ & $853(61.4 \%)$ & 0.216 \\
\hline Symptom-to-first medical contact time [min] & $60.00(30.00,150.00)$ & $80.00(35.00,182.00)$ & $<0.001$ \\
\hline Symptom-to-door time [min] & $194.00(121.00,305.00)$ & $90.00(47.25,191.00)$ & $<0.001$ \\
\hline Door-to-balloon time [min] & $75.00(57.00,102.00)$ & $91.00(67.00,129.00)$ & $<0.001$ \\
\hline Symptom-to-balloon time [min] & $283.00(200.00,406.00)$ & $205.00(141.00,315.00)$ & $<0.001$ \\
\hline Killip class: & & & 0.831 \\
\hline 1 & $1590(74.4 \%)$ & $1015(74.3 \%)$ & \\
\hline ॥ & $270(12.6 \%)$ & $184(13.5 \%)$ & \\
\hline III & $123(5.8 \%)$ & $72(5.3 \%)$ & \\
\hline IV & $154(7.2 \%)$ & $95(7.0 \%)$ & \\
\hline Maximum troponin I [ng/mL] & $72.52 \pm 99.91$ & $75.71 \pm 339.52$ & 0.771 \\
\hline B-type natriuretic peptide $[\mathrm{pg} / \mathrm{mL}]$ & $357.55 \pm 51,908.35$ & $415.88 \pm 1,364.86$ & 0.615 \\
\hline Serum creatinine $[\mathrm{mg} / \mathrm{dL}]$ & $1.07 \pm 0.73$ & $1.12 \pm 0.85$ & 0.028 \\
\hline Left ventricular ejection fraction [\%] & $51.30 \pm 17.56$ & $51.10 \pm 11.79$ & 0.721 \\
\hline
\end{tabular}

Table 2. Angiographic and procedural characteristics.

\begin{tabular}{|c|c|c|c|}
\hline Variable & $\begin{array}{l}\text { Transfer group } \\
(n=2,176)\end{array}$ & $\begin{array}{l}\text { Direct-arrival group } \\
\qquad(\mathrm{n}=1,400)\end{array}$ & $\mathbf{P}$ \\
\hline Extent of CAD: & & & 0.275 \\
\hline 1 vessel disease & $1084(50.1 \%)$ & $669(48.1 \%)$ & \\
\hline 2 vessel disease & $627(29.0 \%)$ & $394(28.3 \%)$ & \\
\hline 3 vessel disease & $413(19.1 \%)$ & $303(21.8 \%)$ & \\
\hline Left main disease & $38(1.8 \%)$ & $25(1.8 \%)$ & \\
\hline Culprit lesion: & & & 0.368 \\
\hline Left anterior descending & $1107(51.1 \%)$ & $677(48.7 \%)$ & \\
\hline Left circumflex & $212(9.8 \%)$ & $150(10.8 \%)$ & \\
\hline Right coronary artery & $826(38.2 \%)$ & $545(39.2 \%)$ & \\
\hline Left main & $20(0.9 \%)$ & $18(1.3 \%)$ & \\
\hline Baseline TIMI flow grade: & & & 0.01 \\
\hline 0 & $1388(64.9 \%)$ & $825(60.1 \%)$ & \\
\hline 1 & $178(8.3 \%)$ & $149(10.9 \%)$ & \\
\hline 2 & $295(13.8 \%)$ & $201(14.7 \%$ & \\
\hline 3 & $279(13.0 \%)$ & $197(14.4 \%)$ & \\
\hline Stent implanted & $2038(93.7 \%)$ & $1304(93.1 \%)$ & 0.828 \\
\hline Drug-eluting stent & $2028(94.1 \%)$ & $1286(92.9 \%)$ & 0.136 \\
\hline Number of stents implanted per patients & $1.39(0.73 \%)$ & $1.35(0.69 \%)$ & 0.105 \\
\hline Procedural success & $2068(96.0 \%)$ & $1329(96.2 \%)$ & 0.685 \\
\hline
\end{tabular}

CAD - coronary artery disease; TIMI — Thrombolysis in Myocardial Infarction 
Table 3. Clinical outcomes.

\begin{tabular}{|c|c|c|c|}
\hline Variable & $\begin{array}{l}\text { Transfer group } \\
\quad(n=2176)\end{array}$ & $\begin{array}{l}\text { Direct-arrival group } \\
(\mathrm{n}=1400)\end{array}$ & $\mathbf{P}$ \\
\hline \multicolumn{4}{|l|}{ In-hospital } \\
\hline Death & $103(5.1 \%)$ & $51(3.9 \%)$ & 0.980 \\
\hline Non-fatal MI & $5(0.2 \%)$ & $1(0.1 \%)$ & 0.252 \\
\hline Revascularization: & $13(0.6 \%)$ & $9(0.6 \%)$ & 0.865 \\
\hline $\mathrm{Re}-\mathrm{PCl}$ & $5(0.2 \%)$ & $0(0.0 \%)$ & 0.073 \\
\hline CABG & $8(0.4 \%)$ & $9(0.6 \%)$ & 0.243 \\
\hline MACE & $117(5.4 \%)$ & $67(4.8 \%)$ & 0.435 \\
\hline \multicolumn{4}{|l|}{ 1-month } \\
\hline Death & $118(5.9 \%)$ & $67(5.1 \%)$ & 0.349 \\
\hline Non-fatal MI & $13(0.6 \%)$ & $9(0.7 \%)$ & 0.891 \\
\hline Revascularization: & $35(1.6 \%)$ & $26(1.9 \%)$ & 0.575 \\
\hline $\mathrm{Re}-\mathrm{PCl}$ & $23(1.1 \%)$ & $12(0.9 \%)$ & 0.554 \\
\hline CABG & $12(0.6 \%)$ & $14(1.0 \%)$ & 0.123 \\
\hline MACE & $162(7.4 \%)$ & $108(7.7 \%)$ & 0.766 \\
\hline \multicolumn{4}{|l|}{ 6-month } \\
\hline Death & $138(6.9 \%)$ & $82(6.3 \%)$ & 0.487 \\
\hline Non-fatal MI & $16(0.8 \%)$ & $12(0.9 \%)$ & 0.714 \\
\hline Revascularization: & $93(4.3 \%)$ & $70(5.0 \%)$ & 0.310 \\
\hline $\mathrm{Re}-\mathrm{PCl}$ & $80(3.7 \%)$ & $56(4.0 \%)$ & 0.622 \\
\hline CABG & $15(0.7 \%)$ & $15(1.1 \%)$ & 0.221 \\
\hline MACE & $241(11.1 \%)$ & 167 (11.9\%) & 0.433 \\
\hline \multicolumn{4}{|l|}{ 12-month } \\
\hline Death & $172(8.2 \%)$ & $89(6.6 \%)$ & 0.075 \\
\hline Non-fatal MI & $17(0.8 \%)$ & $16(1.2 \%)$ & 0.276 \\
\hline Revascularization: & $126(5.8 \%)$ & $89(6.4 \%)$ & 0.487 \\
\hline $\mathrm{Re}-\mathrm{PCl}$ & $110(5.1 \%)$ & $76(5.4 \%)$ & 0.624 \\
\hline CABG & $18(0.8 \%)$ & $15(1.1 \%)$ & 0.456 \\
\hline MACE & $299(13.7 \%)$ & $194(13.9 \%)$ & 0.922 \\
\hline
\end{tabular}

CABG - coronary artery bypass grafting; MACE — major adverse cardiac events; $\mathrm{MI}$ - myocardial infarction; PCI — percutaneous coronary intervention

Angiographic and procedural characteristics

Angiographic and procedural characteristics are presented in Table 2 . In the transfer and direct arrival groups, $93.7 \%$ and $93.1 \%$ of patients underwent stent implantation ( $\mathrm{p}=0.828)$. Drug-eluting stents were used in both the transfer group (94.1\%) and direct-arrival group $(92.9 \%, \mathrm{p}=0.136)$. The procedural success rate was similar in the transfer and direct-arrival groups $(96.0 \%$ vs. $96.2 \%, \mathrm{p}=0.685)$. Except for the baseline Thrombolysis in Myocardial Infarction (TIMI) flow grade, which showed a statistical difference, the other angiographic and procedural characteristics were similar between the transfer and direct-arrival groups.

\section{Clinical outcomes}

The incidence of MACE during the follow-up period according to time-to-PCI is shown in Table 3. In the transfer and direct-arrival groups, the patients' incidence of composite MACE in the hospital was $5.4 \%$ and $3.9 \%$, respectively $(\mathrm{p}=0.435)$. The incidence of composite MACE, death, non-fatal MI, and revascularization were not statistically different between the groups. Also, there were no significant differences in 1 -month MACE $(7.4 \%$ vs. $7.7 \%, \mathrm{p}=0.766)$, 6 -month MACE $(11.1 \%$ vs. $11.9 \%, \mathrm{p}=0.433)$, and 1 -year MACE $(13.7 \%$ vs. $13.9 \%, \mathrm{p}=0.922)$ in each group. 


\section{Discussion}

We evaluated the relationship between clinical outcomes and transfer to hospitals with PCI capability in STEMI patients. The present study has several major findings. First, compared to the direct-arrival group, the symptom-onset-to-first medical contact time and door-to-balloon time were significantly shorter in the transfer group. However, the symptom onset-to-door time was longer in the transfer group than in the directarrival group. Therefore, the symptom-to-balloon time was significantly longer in the transfer group compared to the direct-arrival group. In the case of transfer, there is time to prepare an appropriate treatment before patient arrival. Therefore, the door-to-balloon time was significantly shorter in the transfer group. Importantly, however, the total death and the composite of MACE did not differ significantly both during hospitalization and after 1 year. Transferring STEMI patients to a PCI-capable hospital even with an acceptable time delay resulted in a modest increase in ischemia time, but did not affect clinical outcomes at 1 -year. Therefore, this study suggests that inter-hospital transfer should be encouraged even with delay for STEMI patients who require primary PCI.

Primary PCI is the best treatment option for reperfusion. Previous studies have shown that primary PCI is superior to fibrinolytic therapy in reducing death, non-fatal reinfarction, and stroke [13-15]. In addition, multiple European studies have demonstrated that transfer of patients to PCI-capable hospitals is associated with lower mortality compared to onsite fibrinolytic therapy [16-18]. These studies asserted that patients with STEMI, even when first admitted to a non-PCI-capable hospital, should be transported to a PCI-capable hospital. Importantly, Widimsky et al. [18] showed that long distance transport from a community hospital to a tertiary PCI center in the acute phase of acute MI is safe. This study was conducted in the Czech Republic. Our study showed similar results, which may be due to the fact that Korea's geographic characteristics may be similar to those of the Czech Republic.

Several studies show that delaying primary PCI increases mortality risk and results in poor clinical outcomes [3, 4]. Pinto et al. [19] showed in a large analysis of STEMI transfer patients that the mortality benefit of primary PCI over on-site fibrinolysis disappears when the PCI-related delay (door-to-balloon time minus door-to-needle time, in matched pairs) was 120 min or longer.
Accordingly, many studies have made an effort to reduce the door-to-balloon time. Some studies suggest skipping local hospitals in favor of direct referral to PCI-capable hospitals via pre-hospital electrocardiogram diagnosis $[8,9]$. This strategy is very effective and shows better clinical outcomes. However, in many situations, emergency medical services do not have well-trained emergency medical services providers and cannot employ this strategy.

Currently, many patients with STEMI are transferred to PCI-capable hospitals. Our study showed that transfer to PCI-capable hospitals with a short delay did not increase the mortality rate. Because of the geographical characteristics in South Korea, the time difference was not significantly different between transfer patients and direct-arrival patients. In the case of countries with more expansive cities, such as America or Europe, there could be different results. Therefore, inter-hospital transfer of STEMI patients should be encouraged even with an acceptable time delay for primary PCI in areas with similar geographic characteristics.

\section{Limitations of the study}

Our study has several limitations. First, this is an observational study using data from a prospectively gathered nationwide registry. Our study was not a randomized control study, and there were significant differences in the baseline characteristics and angiographic characteristics. Second, we only have data on patients with STEMI who presented to the hospital. Data on those who did not present to the hospital, who died at home, or who died during transport to the hospital were not available for analysis. Hence, there is a survival bias that could have confounded our results. Third, in this study, only 1 -year clinical outcomes were followed. More data are needed on long-term outcomes in patients with STEMI.

\section{Conclusions}

According to the KAMIR data, approximately $60 \%$ of patients with STEMI initially arrive at non-PCI capable hospitals and are transferred to PCI-capable hospitals. Transferring STEMI patients to PCI-capable hospitals delays reperfusion time. However, it does not affect clinical outcomes at 1-year. Our results suggest that inter-hospital transfer of STEMI patients should be encouraged even with an acceptable delay for primary PCI in areas with a similar geographic accessibility. 


\section{Acknowledgements}

This work was supported by the Medical Research Center (MRC) Program through the National Research Foundation of Korea (NRF) grant funded by the Government of South Korea (MSIP) (NRF-2015R1A5A2009656).

\section{Conflict of interest: None declared}

\section{References}

1. Fosbol EL, Granger CB, Jollis JG et al. The impact of a statewide pre-hospital STEMI strategy to bypass hospitals without percutaneous coronary intervention capability on treatment times. Circulation, 2013; 127: 604-612. doi: 10.1161/CIRCULATIONAHA.112.118463.

2. Cannon CP, Gibson CM, Lambrew CT et al. Relationship of symptom-onset-to balloon time and door-to-balloon time with mortality in patients undergoing angioplasty for acute myocardial infarction. JAMA, 2000; 283: 2941-2947. doi: 10.1016/j. jjcc.2011.06.001.

3. De Luca G, Suryapranata H, Ottervanger JP, Antman EM. Time delay to treatment and mortality in primary angioplasty for acute myocardial infarction: Every minute of delay counts. Circulation, 2004; 109: 1223-1225. doi: 10.1161/01. CIR.0000121424.76486.20.

4. McNamara RL, Wang Y, Herrin J et al. Effect of door-to-balloon time on mortality in patients with ST-segment elevation myocardial infarction. J Am Coll Cardiol, 2006; 47: 2180-2186. doi: 10.1016/j.jacc.2005.12.072.

5. O'Gara PT, Kushner FG, Ascheim DD et al. 2013 ACCF/AHA guideline for the management of ST-elevation myocardial infarction: A report of the American College of Cardiology Foundation/American Heart Association Task Force on Practice Guidelines. J Am Coll Cardiol, 2013; 61: e78-e140. doi: 10.1016/j. jacc.2012.11.019.

6. Borger MA, Di Mario C, Dickstein K et al. ESC Guidelines for the management of acute myocardial infarction in patients presenting with ST-segment elevation. Eur Heart J, 2012; 33: 2569-2619. doi: 10.1093/eurheartj/ehs215.

7. Jacobs AK, Antman EM, Faxon DP, Gregory T, Solis P. Development of systems of care for ST-elevation myocardial infarction patients: executive summary. Circulation, 2007; 116: 217-230. doi: 10.1161/CIRCULATIONAHA.107.184043.

8. Le May MR, So DY, Dionne R et al. A citywide protocol for primary PCI in ST-segment elevation myocardial infarction. N Engl J Med, 2008; 358: 231-240. doi: 10.1056/NEJMoa 073102 .

9. Sørensen JT, Terkelsen CJ, Nørgaard BL et al. Urban and rural implementation of pre-hospital diagnosis and direct referral for primary percutaneous coronary intervention in patients with acute ST-elevation myocardial infarction. Eur Heart J, 2011; 32: 430-436. doi: 10.1093/eurheartj/ehq437.

10. Chen K-Y, Rha S-W, Li Y-J et al. Triple versus dual antiplatelet therapy in patients with acute ST-segment elevation myocardial infarction undergoing primary percutaneous coronary intervention. Circulation, 2009; 119: 3207-3214. doi: 10.1161/CIRCULATIONAHA.108.822791.

11. Kang S-H, Suh J-W, Yoon C-H et al. Sex differences in management and mortality of patients with ST-elevation myocardial infarction (from the Korean Acute Myocardial Infarction National Registry). Am J Cardiol, 2012; 109: 787-793. doi: 10.1016 /j.amjcard.2011.11.006.

12. Thygesen K, Alpert JS, Jaffe AS et.al. Third universal definition of myocardial infarction. Eur Heart J, 2012; 33: 2551-2567. doi: 10.1093/eurhearti/ehs184.

13. The Global Use of Strategies to Open Occluded Coronary Arteries in Acute Coronary Syndromes (GUSTO IIb) Angioplasty Substudy Investigators. A clinical trial comparing primary coronary angioplasty with tissue plasminogen activator for acute myocardial infarction. N Engl J Med, 1997; 336: 1621-1628. doi: 10.1056/NEJM199706053362301.

14. Aversano T1, Aversano LT, Passamani E et al. Thrombolytic therapy vs. primary percutaneous coronary intervention for myocardial infarction in patients presenting to hospitals without on-site cardiac surgery: A randomized controlled trial. JAMA, 2002; 287: 1943-1951. doi: 10.1001/jama.287.15.1943.

15. Keeley EC1, Boura JA, Grines CL. Primary angioplasty versus intravenous thrombolytic therapy for acute myocardial infarction: A quantitative review of 23 randomised trials. Lancet, 2003; 361: 13-20. doi: 10.1016/S0140-6736(03)12113-7.

16. Andersen HR, Nielsen TT, Rasmussen $\mathrm{K}$ et al. A comparison of coronary angioplasty with fibrinolytic therapy in acute myocardial infarction. N Engl J Med, 2003; 349: 733-742. doi: 10.1056/ NEJMoa025142.

17. Widimský P, Groch L, Zelízko M, Aschermann M, Bednár F, Suryapranata H. Multicentre randomized trial comparing transport to primary angioplasty vs immediate thrombolysis vs combined strategy for patients with acute myocardial infarction presenting to a community hospital without a catheterization laboratory. The PRAGUE study. Eur Heart J, 2000; 21: 823-831. doi: 10.1053/euhj.1999.1993.

18. Widimský P1, Budesínský T, Vorác D et al. Long distance transport for primary angioplasty vs immediate thrombolysis in acute myocardial infarction. Final results of the randomized national multicentre trial: PRAGUE-2. Eur Heart J, 2003; 24: 94-104. doi: 10.1016/S0195-668X(02)00468-2.

19. Pinto DS, Frederick PD, Chakrabarti AK et al. Benefit of transferring ST-segment elevation myocardial infarction patients for percutaneous coronary intervention compared with administration of onsite fibrinolytic declines as delays increase. Circulation, 2011; 124: 2512-2521. doi: 10.1161/CIRCULATIONAHA.111.018549. 